\title{
Recognition of Normal and Abnormal Cells through SERS-Active FIB-Fabricated Au Nanoneedle Array Structure
}

\author{
Kundan Sivashanmugan ${ }^{1}$, Jiunn-Der Liao ${ }^{1,}$ 2, 3*, Pei-Lin Shao ${ }^{1}$ \\ 1Department of Materials Science and Engineering, National Cheng Kung University, 1 University Road, \\ Tainan 70101, Taiwan. \\ ${ }^{2}$ Center for Micro/Nano Science and Technology, National Cheng Kung University, 1 University Road, Tainan \\ 70101, Taiwan. \\ 3Medical Device Innovation Center, National Cheng Kung University, 1 University Road, Tainan 70101, \\ Taiwan.
}

* Corresponding author. Tel: +886-6-2757575 ext. 62971; email: jdliao@mail.ncku.edu.tw Manuscript submitted November 10, 2014; accepted December 31, 2014.

doi: 10.17706/ijbbb.2015.5.1.54-61

\begin{abstract}
Au nanoneedle arrays were fabricated using a focused ion beam ( $\left.f i b A u_{-} N N\right)$. With rhodamine 6G used as the probe molecule on the optimized Surface-enhanced Raman scattering (SERS) substrate, an enhancement of 7 orders of magnitude was obtained at low concentration $\left(10^{-5} \mathrm{M}\right)$. Moreover, a strong electromagnetic field effect is generated in and around these NNs, creating localized surface plasmon resonance. In the biological assessment, the optimized fibAu_NN substrate was able to distinguish cancer and normal cells. The SERS effect was extensively increased at incident laser interacted area of cells and sharp NNs surfaces.
\end{abstract}

Key words: Au nanoneedle, focused ion beam, electromagnetic field, cells.

\section{Introduction}

Fluorescence imaging technology with fluorescence-based materials are widely applied to cellular imaging and biomedical application [1]; whoever, they have intrinsic problems, such as imaging and diagnostics techniques often lack sensitivity and selectivity to the environmental conditions of biological samples [2], [3]. In particularly, metal and inorganic metal nanoparticles (NP) have been extensively used as fluorescent labeling agents for biological diagnostics, which have excellent sensitivity [4]. However, particular labeling techniques are required, low toxicity and high water solubility in vivo biomedical application. Surface-enhanced Raman scattering (SERS) is an ultrasensitive technique that greatly enhances Raman signals of single molecule level [5]. SERS can provide characteristic spectroscopic fingerprints of clinical macromolecules such as a variety of single cells, including cancer cells [6]. Recently, few research groups have already reported the applications of SERS for clinical diagnostics by using labeling NPs agents [6], [7]. The labeling NPs based SERS application technology, especially for cellular imaging or biomedical diagnostics, still has a strong need for improvement in its sensitivity, selectivity and good biocompatibility. In addition, there are two typical disadvantages of SERS that as relatively weak Raman signals when laser polarized in analyte molecules interact with a metal surface and another drawback is long data collection time for spectral acquisition that could either damage the biologic sample. The target specie damage can be reduced, and the strong localized surface plasmon resonance (LSPR) active SESR-substrate was increased 
within the short time interaction with target species.

The enhancement of the Raman signal intensity depends strongly on surface plasmons that give increase in the Raman scattering [8]. Currently, the present authors have made highly reproducible SERS-active substrates with $\mathrm{Au}$ and $\mathrm{Au} / \mathrm{Ag}$ multilayer nanorod (NR) arrays via focused-ion-beam (FIB) technology [9]. The NR arrays SERS substrate can induce a LSPR effect due to the availability of interface gap effect, multiple edges and a small curvature. The macromolecule size samples (i.e., cells) were required strong LSPR substrate to acquire the Raman information from target species. However, the Raman scattering characteristics strongly depended on the geometry of nanostructures [10]. In this work, Au nanoneedle (NN) arrays were fabricated using a focused ion beam (FIB). With rhodamine 6G (R6G) as the probe molecule on the optimized SERS active substrate, the optimized Au NNs arrays were able to distinguish normal and cancer cells.

\section{Experimental Section}

\subsection{Fabrication of Au Nanoneedle Arrays}

An Au was deposited on a single-crystal silicon (100) wafer primed with a $5 \mathrm{~nm}$ thick adhesion layer of titanium by an e-beam evaporator (VT1-10CE, ULVAC, Taiwan). An average roughness of $\approx 1.42 \mathrm{~nm}$ and X-ray diffraction pattern (111) were obtained [9], [10]. The optimal thickness of Au layer was kept $\approx 350 \mathrm{~nm}$. NNs patterns were designed using CorelDRAW software. The designs were implemented using a focused ion beam (SMI 3050, SII Nanotechnology, Japan). By adjusting the current and etching time, Au NN arrays (fibAu_NN) with distinct parameters were fabricated. The pattern size was about $30 \times 30 \mu \mathrm{m}$ with beam conditions of $30 \mathrm{kV}$ acceleration voltage with UFine , $0.07 \mu \mathrm{m}$ depth, $10 \mathrm{pA}$ aperture, $70 \mu \mathrm{sec}$ dwell time, $+0.56 \mathrm{OL}$ fine, and 8 -image scale with $50 \%$ overlap. As illustrated in Fig. 1, the as-prepared fibAu_NN samples NN_1 to NN_3 was set based on geometric factors - the NNs tip-to-tip distance $\left(D_{t-\mathrm{t}}\right)$ varies from $\approx 85$ to $\approx 139 \mathrm{~nm}$. Field-emission scanning electron microscopy (FE-SEM, JSM-7001, JEOL, Japan) was used to analyze the morphologies of as-prepared fibAu_NN samples.

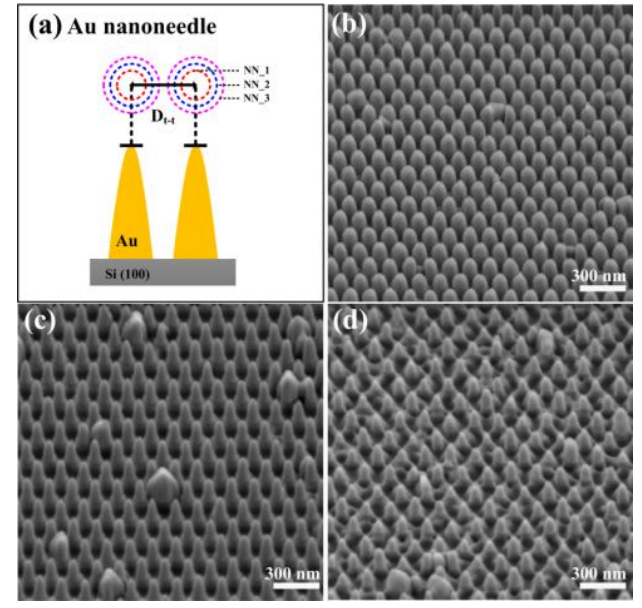

Fig. 1. Schematic illustration of as-prepared fibAu_NN samples (a). FE-SEM side-view image of the as-fabricated $f i b A u \_N N$ samples NN_1 (b), NN_2 (c), and NN_3 (d).

\subsection{Molecular Probes for the Evaluation of Enhancement Factor}

Rhodamine 6G (R6G), used as the molecular probe, was prepared and diluted in aqueous solution to a concentration of $10^{-5} \mathrm{M}$. To verify the EF of fibAu_NN substrates with molecular-probe-containing, the solution was covered with a glass slide and then immediately measured using a confocal microscopy Raman spectrometer (inVia Raman microscope, Renishaw, United Kingdom) using a laser at $785 \mathrm{~nm}$ with a magnification of $50 \times$. The samples with the molecular-probe-containing solution were scanned with an 
integration time of $10 \mathrm{~s}$ over an area of $1 \mu \mathrm{m} \times 1 \mu \mathrm{m}$ (the size of the laser spot was $\sim 1 \mu \mathrm{m}$ ). Before each batch, the Raman shift was calibrated using a signal of $520 \mathrm{~cm}^{-1}$ with the absolute intensity from a standard silicon wafer. The EF measurement was estimated according to the standard equation [9]-[11]:

$$
E F=\frac{I_{\text {sers }}}{I_{\text {bulk }}} \times \frac{N_{\text {bulk }}}{N_{\text {sers }}}
$$

Where $I_{\text {sers }}$ and $I_{\text {bulk }}$ are SERS and normal Raman scattering intensities, respectively; $N_{\text {sers }}$ and $N_{\text {bulk }}$ are the numbers of molecules contributing to the inelastic scattering intensity respectively evaluated by SERS and normal Raman scattering measurements.

\subsection{Biocompatibility Studies and Raman Detection}

The biocompatibility testing and Raman detection of the as-fabricated fibAu_NN samples were mainly performed using the tumor cancer (i.e., FaDu) and live/dead 3T3 (i.e., fibroblasts (FB)) cell staining protocol. FaDu and FB cell lines were preserved in Dulbecco's Modified Eagle's Medium (DMEM) and $10 \mathrm{ml}$ of 10,000 units/ml penicillin - 10,000 $\mu \mathrm{g} / \mathrm{ml}$ streptomycin (Sigma, St Louis, MO, USA). Before the experiments, FaDus and FBs cells were washed with phosphate-buffered saline (PBS) and detached with trypsine (Gibco, Invitrogen, CA, USA). For the MTS assay, FaDu and FB cells were seeded near confluence $(8,000$ cells $/$ well $=$ $2.7 \times 10^{5}$ cells $/ \mathrm{ml}$ ) on 48 -well plates (Nunc, Thermal Scientific, Denmark). The cells were then cultured in a complete medium, maintained at $37{ }^{\circ} \mathrm{C}$ under $5 \% \mathrm{CO}_{2}$ in an incubator for $24 \mathrm{~h}$. In the cell coverage model, $\mathrm{FaDu}$ and FB cells were placed consistently in a 24-well plate containing fibAu_NN samples (Nunc, Thermal Scientific), with $2.7 \times 10^{5}$ cells $/ \mathrm{ml}$ in a complete medium, and maintained at $37{ }^{\circ} \mathrm{C}$ under $5 \% \mathrm{CO}_{2}$ for $24 \mathrm{~h}$. FB and FaDu cells at high density on samples were prepared for Raman analysis with $785 \mathrm{~nm}$ wavelength.
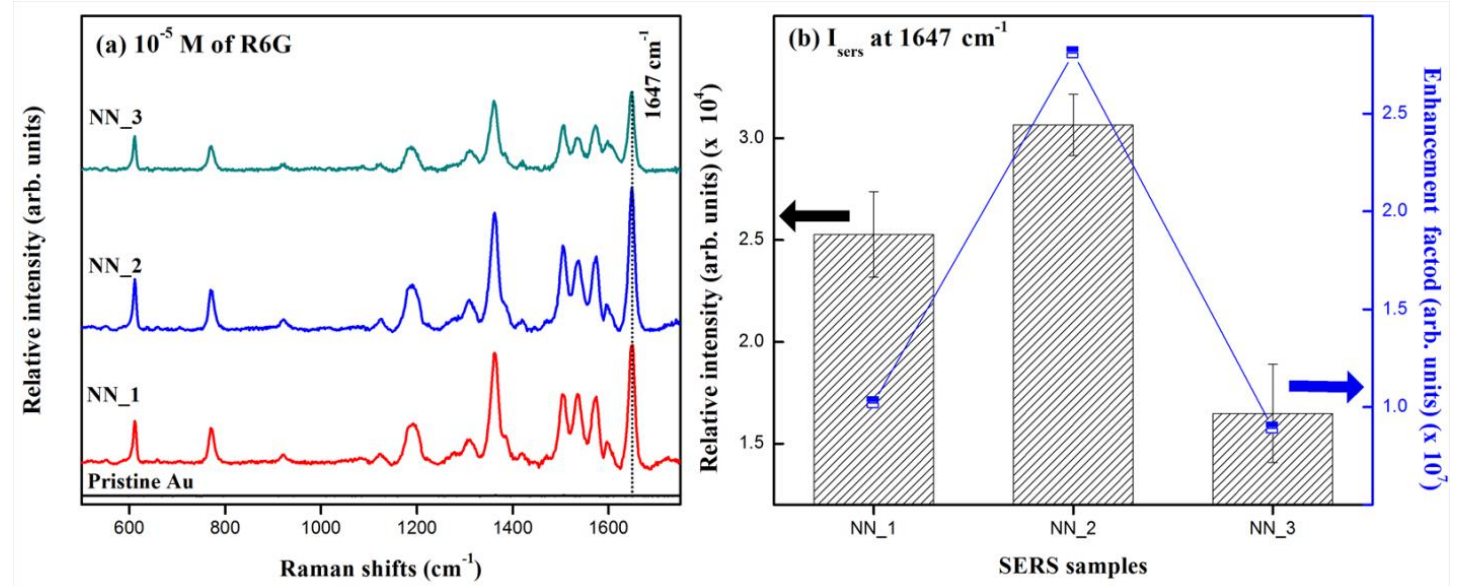

Fig. 2. (a) Raman-active peaks of $10^{-5} \mathrm{M}$ R6G molecules on fibAu_NN samples NN_1, NN_2, and NN_3 examined at Raman laser wavelength of $785 \mathrm{~nm}$. (b) Enhancement factors and relative Raman intensities for samples NN_1 to NN_3. R6G $\left(10^{-5} \mathrm{M}\right)$ at $1647 \mathrm{~cm}^{-1}$ were used as the index for the relation of relative Raman intensities with respect to samples NN_1 to NN_3.

\section{Results and Discussion}

\subsection{Characterization and Optimization of fibAu_NNs as SERS-Active Substrates}

Fig. 1(b-d) shows the side-view FE-SEM images of as-fabricated fibAu_NN samples NN_1 to NN_3. The NNs length and diameter of the as-fabricated fibAu_NN samples NN_1 to NN_3 were 330, 240, $170 \mathrm{~nm}$ (i.e., length) and 61, 25, 20 (i.e., diameter) $\mathrm{nm}$, respectively. The NNs shape and size were maintained by 
adjusting the working current and etching time during FIB fabrication. The varied $D_{\mathrm{t}-\mathrm{t}}$ distances simultaneously changed with the formation of sharp NNs surface. In particularly, sharp NNs surface was significantly producing the SPR effect [10], [12].

R6G solution $\left(10^{-5} \mathrm{M}\right)$ was used to determine the SERS effect for fibAu_NN samples NN_1 to NN_3 via Raman spectroscopy using a diode laser with a wavelength of $785 \mathrm{~nm}$. The $f i b A u_{-}$NN samples NN_1 to NN_3 exhibited strong enhancement of the vibrational modes, as shown in Fig. 2(a). The SERS spectrum of R6G contains most of its characteristic peaks ascribed to ring C-C stretching modes, the most intense of which appear at a Raman shift of about $1649 \mathrm{~cm}^{-1}$ on samples NN_1 to NN_3. According to Eq. (1), the EF values of samples NN_1 to NN_3 reached 107, as shown in Fig. 2(b). The ring C-C stretching $\left(1649 \mathrm{~cm}^{-1}\right)$ band intensity and EF of sample NN_2 were the most strongly enhanced with compare to the other sample [9]-[11]. However, the EM field effect was strongly generated in and around these large NNs, creating LSPR effect. In Fig. 3, Raman peak intensities at $1649 \mathrm{~cm}^{-1}$ for R6G with concentrations of 10-7, $10^{-9}$, and 10-12 M upon optimized samples NN_2 were furthermore compared. The samples NN_2 significantly increased SERS intensity of R6G with three different concentrations. As a consequence, the NNs size uniformly formed and let to the enhance SERS effect.

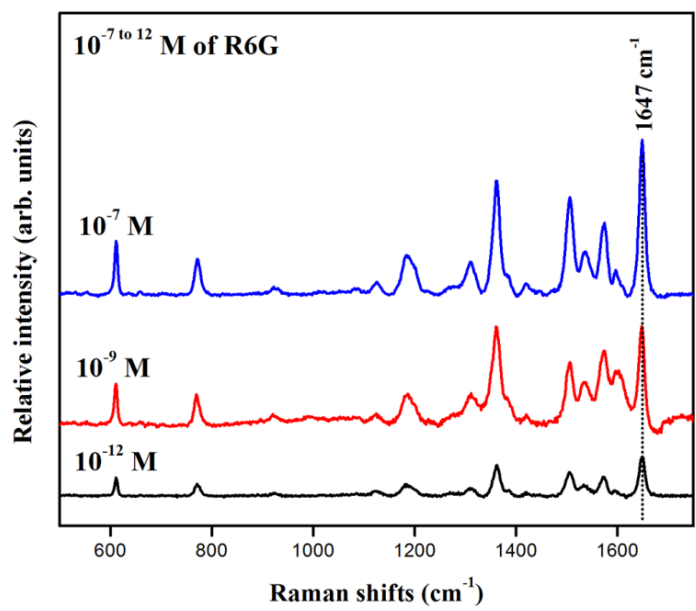

Fig. 3. Raman-active peaks of $10^{-12}$ to -7 M R6G molecules on optimized fibAu_Ns sample NN_2 examined at Raman laser wavelength of $785 \mathrm{~nm}$.

\subsection{Biocompatibility and Raman Studies of FB and FaDu Cells on fibAu_NN Sample}

The presence of fibAu_NN samples NN_1 to NN_3 samples with cell morphology and viability (FB and FaDu-cells) were studied, as shown in Fig. 4(a-b). Fig. 4(a) shows the high viabilities of FB and FaDu cells on samples NN_1 to NN_3. The fluorescence images of live/dead cell staining protocol are shown in Fig. 4(b), with pristine $\mathrm{Au}$ substrate used as the reference. In addition, the NN_2 samples thus support continuous cellular growth for upto $24 \mathrm{~h}$. Among those studies, the fibAu_NN samples NN_1 to NN_3 were the most biocompatible with FaDu cell with compare to the FB cells. However, the FB cells are more sensitive in cell culartal enviroment.

The SERS spectra for the control, NN_1, and NN_2 samples with FB and FaDu cells were evaluated at a laser wavelength of $785 \mathrm{~nm}$ and 10\% power, as shown in Fig. 4(c)-(d). A pristine Au, control, and NN_1 were used as the detection reference for SERS measurement. The average spectra of FB and FaDu- cells have prominent spectral peaks at $617,795,1000,1031-1036,1154 \sim 1156,1190 \sim 1196,1326,1449 \sim 1150,1583$, and 1602 1603 $\mathrm{cm}^{-1}$ [7], [13]-[16]. These spectral features arise from the molecular vibrations of cell components such as lipids, nucleic acids, and protein, as listed in Table 1. The Raman spectra of FB and FaDu-cells on sample NN_2 could be strongly assigned to the symmetric ring breathing mode of 
phenylalanine (1031 and $1000 \mathrm{~cm}^{-1}$ ). The FaDu cells showed lower Raman intensities of nucleic acids, as indicated in the heights at 795, 1000,1036, 1156, 1190, 1450, 1583, and $1603 \mathrm{~cm}^{-1}$ with compare to the FB cell SERS spectra, Besides the peaks mentioned above, the FaDu cells also showed lower peak heights in the $1603 \mathrm{~cm}^{-1}$ band, which corresponded to the $\mathrm{C}=\mathrm{C}$ stretching mode of tyrosine and tryptophan [13]-[16]. The reduction in the Raman bands observed suggested that the amount of nucleic acid, protein and lipid could be lower in FaDu cells. Notably, the result of SERS might be induced by the combined NNs LSPR polarization and chemical effect (Fig. 5). In the case, chemical effect occurred at the interface of cells and the surfaces of NNs area [9], [10], [17]. In this study, we believed that the SERS spectra of sample NN_2 could be clearly distinguished between FB and FaDu-cells.
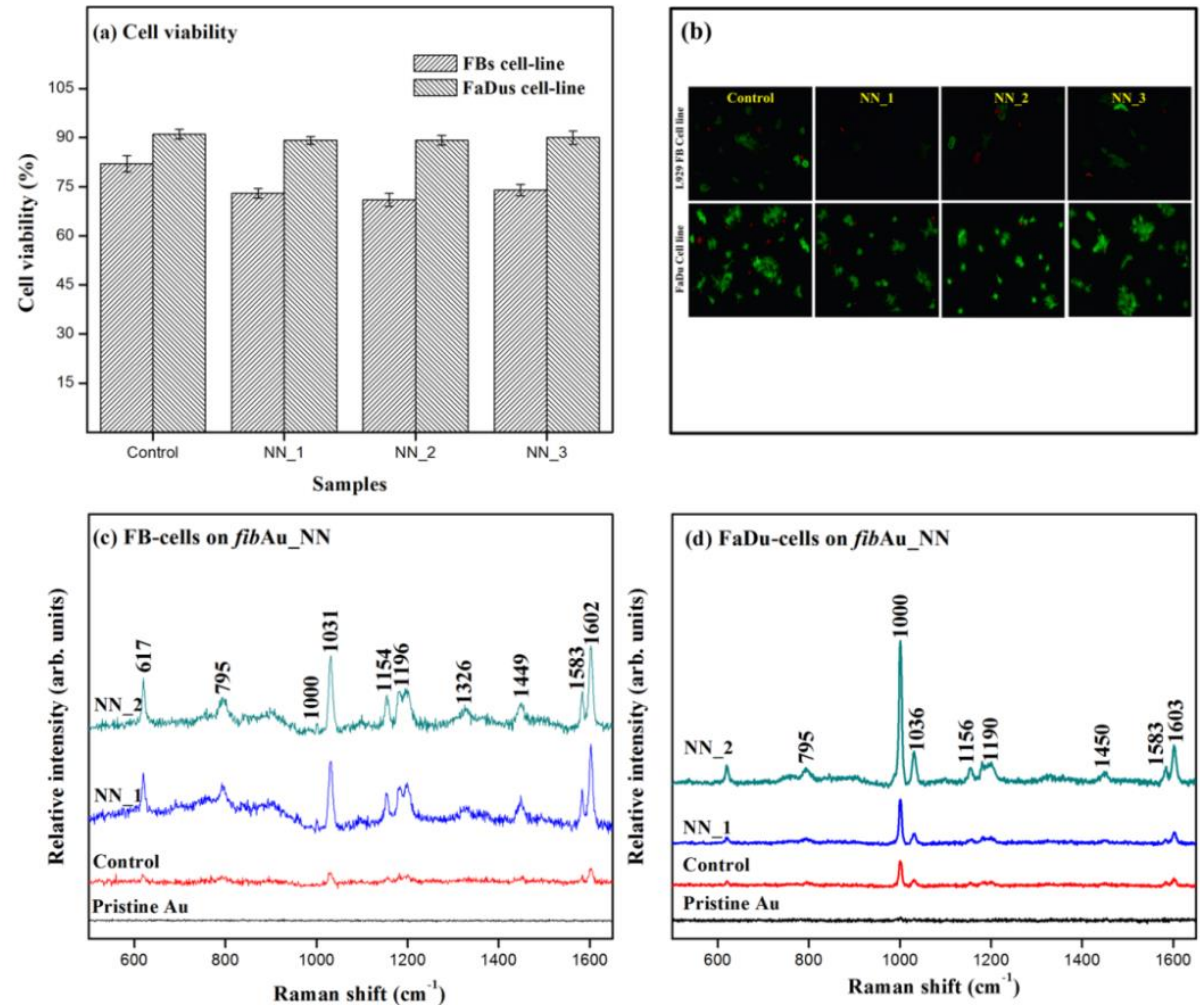

Fig. 4. (a) Cell viability (\%), (b) optical images of FB and FaDu cells coated on fibAu_NN sample NN_1 to NN_2 evaluated by MTT assay analysis compared to the control group. SERS spectra for (c) FB and (d) FaDu cells on samples NN_1 and NN_2 examined at 785 nm Raman laser.

Table 1. Assignment of Raman Bands for FBs and FaDus Cells in SERS Using Au NNs Samples

\begin{tabular}{ll} 
SERS & Assignment \\
\hline 617 & $\begin{array}{l}\text { Phenylalanine } \\
\text { Cytosine/uracil ring breathing } \\
\text { (nucleotide) } \\
\text { Symmetric ring breathing mode } \\
\text { of phenylalanine } \\
\text { Phenylalanine }\end{array}$ \\
1000 & C-C ( \& C-N) stretch of proteins \\
$1031 \sim 1036$ & (also caroteneoids) \\
1154 & Cytosine, guanine \\
1196 & Polynucleotide chain \\
1326 & (DNA-purine bases) \\
1449 & CH2 bending mode of proteins \\
1583 & Proteins \\
1602 & C=C stretching mode of tyrosine \\
& and tryptophan \\
\hline
\end{tabular}




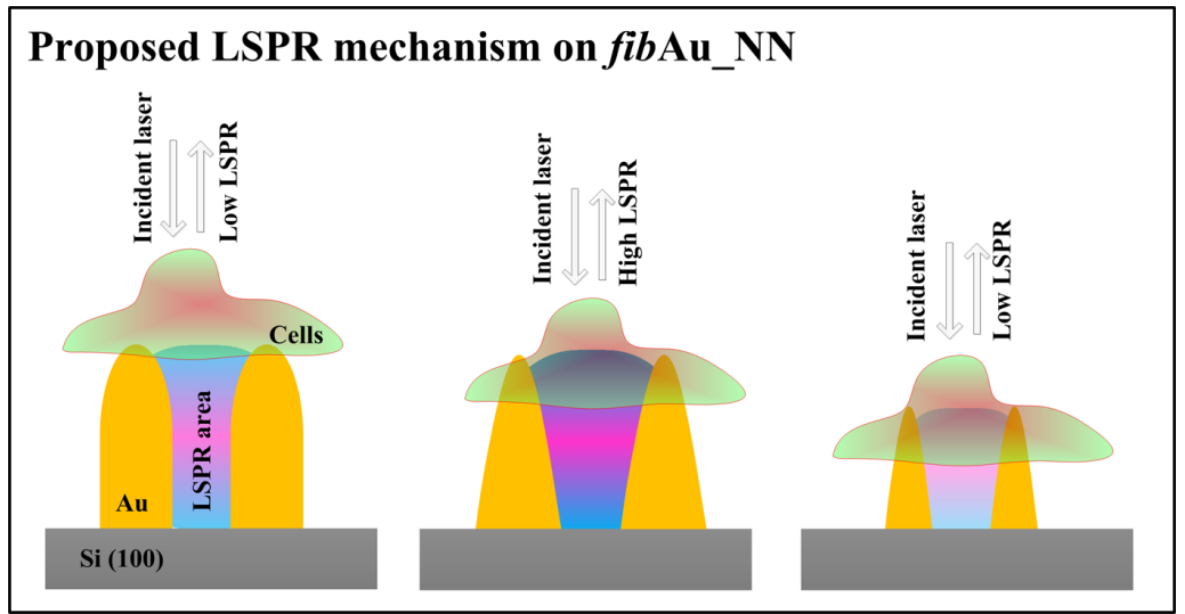

Fig. 5. Brief description of proposed LSPR mechanism from fibAu_NN.

\section{Conclusion}

The fibAu_NNs array was fabricated by using FIB method. The as-prepared fibAu_NN substrate served as an excellent SERS substrate with R6G as the molecular probe, an enhancement of 7 orders of magnitude was obtained at low concentration $\left(10^{-5} \mathrm{M}\right)$. A strong LSPR was generated in and around these NNs, creating large local EM field. Experimental results show that the SERS spectra obtained using fibAu_NNs substrate can be used for detect specific cancer and normal cells.

\section{Acknowledgment}

This work was financially supported by the Ministry of Science and Technology of Taiwan under grant NSC-100-2221-E-006-025-MY3 and the Medical Device Innovation Center of National Cheng Kung University under grant D102-21007.

\section{References}

[1] Guo, X., Guo, Z., Jin, Y., Liu, Z., Zhang, W., Huang, D., et al. (2012). Silver-gold core-shell nanoparticles containing methylene blue as SERS labels for probing and imaging of live cells. Microchim. Acta, 178, 229.

[2] Luo, Z. H., Chen, K., Lu, D. L., Han, H. Y., Zou, M. Q., et al. (2011). Synthesis of paminothiophenol-embedded gold/silver core-shell nanostructures as novel SERS tags for biosensing applications. Microchim. Acta, 173(1-2), 149.

[3] Luo, Z., Fu, T., Chen, K., Han, H., Zou, M., et al. (2011). Synthesis of multibranched gold nanoparticles by reduction of tetrachloroauric acid with Tris base, and their application to SERS and cellular imaging. Microchim. Acta, 175(1-2), 55.

[4] Amano, Y., Cheng, Q., et al. (2005). Detection of influenza virus: Traditional approaches and development of biosensors. Anal. Bioanal. Chem., 381, 156.

[5] Janina, K., Harald, K., Burghardt, W., Katrin, K., et al. (2010). Following the Dynamics of pH in Endosomes of Live Cells with SERS Nanosensors. J. Phys. Chem. C, 114, 7421.

[6] McEwen, G. D., Wu, Y., Tang, M., Qi, X., Xiao, Z., Baker, S. M., et al. (2013). Subcellular spectroscopic markers, topography and nanomechanics of human lung cancer and breast cancer cells examined by combined confocal Raman microspectroscopy and atomic force microscopy. Analyst, 138, 787. 
[7] Feng, S., Chen, R., Lin, J., Pan, J., Chena, G., Li, Y., et al. (2010). Nasopharyngeal cancer detection based on blood plasma surface-enhanced Raman spectroscopy and multivariate analysis. Biosen. Bioelec., 25,2414

[8] Campion, A., Kambhampati, P., et al. (1998). Surface-enhanced Raman scattering. Chem. Soc. Rev., 27, 241.

[9] Sivashanmugan, K., Liao, J. D., You, J. W., Wu, C. L., et al. (2013). Focused ion beam fabricated Au/Ag multilayered nanorod array as SERS-active substrate for virus strain detection. Sens. Act. B: Chem., $181,361$.

[10] Sivashanmugan, K., Liao, J. D., Liu, B. H., Yao, C. K., et al. (2013). Focused-ion-beam-fabricated Au nanorods coupled with Ag nanoparticles used as SERS-active substrate for analyzing trace melamine constituents in solution. Anal. Chim. Acta, 800, 56.

[11] He, L. L., Kim, N. J., Li, H., Hu, Z. Q., Lin, M. S., et al. (2008). Use of a fractal-like gold nanostructure in surface-enhanced raman spectroscopy for detection of selected food contaminants. J. Agric. Food Chem., 56, 9843.

[12] Lordan, F., Damm, S., Kennedy, E., Mallon, C., Forster, R. J., Keyes, T. E., et al. (2013). The effect of Ag nanoparticles on surface-enhanced luminescence from $\mathrm{Au}$ nanovoid arrays. Plasmonics, 8, 1567-1575.

[13] Zhong, H., Xu, J. J., Chen, H. Y., et al. (2004). Determination of trace proteins by Rayleigh light scattering technique with indophenol blue. Microchim Acta, 148, 99.

[14] Salman, A., Shufan, E., Zeiri, L., Huleihel, M., et al. (2013). Detection and identification of cancerous murine fibroblasts, transformed by murine sarcoma virus in culture, using Raman spectroscopy and advanced statistical methods. Biochimica et Biophysica Acta, 1830, 2720.

[15] Yao, H. L., Tao, Z. H., Ai, M., Peng, L. X., Wang, G. W., He, B. J., \& Li, Y. Q. (2009). Raman spectroscopic analysis of apoptosis of single human gastric cancer cells. Vibration. Spectro., 50,193-197.

[16] Xie, W., Su, L., Shen, A., Maternyc, A., Hua, J., et al. (2011). Application of surface-enhanced Raman scattering in cell analysis. J. Raman Spectrosc., 42, 1248.

[17] Sigle, D. O., Perkins, E., Baumberg, J. J., Mahajan, S., et al. (2013). Reproducible deep-UV SERRS on aluminum nanovoids. J. Phys. Chem. Lett., 4, 1449.

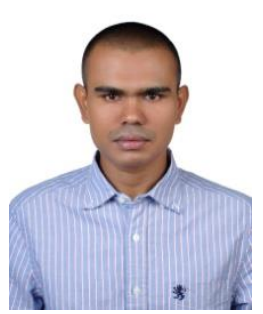

Kundan Sivashanmugan is currently pursuing his $\mathrm{PhD}$ degree in the Department of Materials Science and Engineering (MSE), National Cheng Kung University (NCKU), Tainan, Taiwan. He received his M.Sc. in physics and M.Phil. in nanoscience and technology degrees at Bharathiar University, Coimbatore, India in 2009 and 2011, respectively. His current research interest is focused on the fabrication of SERS-active substrates as a fast-screening detection platform for food contamination and viruses using a focused ion beam (FIB) technique.

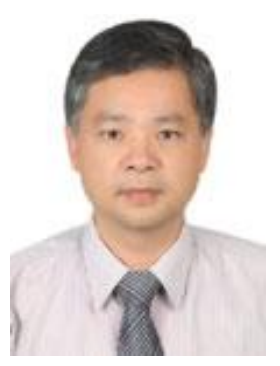

Jiunn-Der Liao is currently a distinguished professor in the Department of MSE, NCKU, Tainan, Taiwan. He obtained his B.S. degree at the same department in 1984, M.S. degrees at K.U. Leuven (Belgium) in 1990 and 1991, respectively, and Ph.D. degree at ENS Mines (France) in 1994. He also worked at University of Heidelberg (Germany) as a research fellow from 1995 to 1996 and Chung Yuan Christian University (Taiwan) as an associate professor from 1996 to 2002. His current research interests are focused on (1) mechanics of biomaterials, e.g., tissue engineering, scaffold materials, cell-surface 
interactions, nano-indentation, mechanical transduction; (2) plasma chemistry and plasma processing, e.g., plasma generation, plasma diagnoses, plasma physics and chemistry, metal vapor vacuum arc, plasma ion immersion; (3) nano-fabrication and nano-characterization, e.g., FIB-based nano-fabrication, micro-contact imprinting, and synchrotron-based and laboratory-based high-resolution analyses.

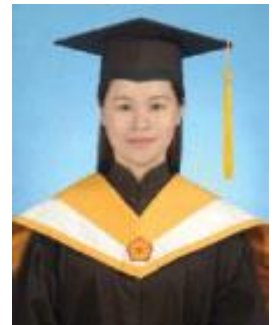

Pei-Lin Shao obtained her M.S. degree in basic medical sciences from Kaohsiung Medical University (KMU, Kaohsiung, Taiwan) in 2003. She obtained her Ph.D. in Institute of Medicine also from KMU in 2011. Her Ph.D. topic was focused on the role of shock wave therapy in attenuating intima injury-induced vascular remodeling. Her current research topic is focused on the advanced wound management. 